\title{
KOSTANT CONVEXITY FOR AFFINE BUILDINGS
}

\author{
PETRA HITZELBERGER \\ FEBRUARY 2008
}

\begin{abstract}
We prove an analogue of Kostants convexity theorem for thick affine buildings and give an application for groups with affine $B N$-pair. Recall that there are two natural retractions of the affine building onto a fixed apartment $A$ : The retraction $r$ centered at an alcove in $A$ and the retraction $\rho$ centered at a chamber in the spherical building at infinity. We prove that for each special vertex $X \in A$ the set $\rho\left(r^{-1}(W . x)\right)$ is a certain convex hull of $W . x$. The proof can be reduced to a problem in Coxeter complexes and heavily relies on a character formula for highest weight representations of algebraic groups.
\end{abstract}

\section{INTRODUCTION}

Kostant Kos73 proved a convexity theorem for symmetric spaces, generalizing a well known theorem of Schur Sch23. Let $G / K$ be a symmetric space and $\mathfrak{g}=\mathfrak{k} \oplus \mathfrak{p}$ the Cartan decomposition of the associated Lie algebra. Let $T$ be a maximal flat of $G / K$ and $W=N_{K}(T) / Z_{K}(T)$ the spherical Weyl group of $G / K$ which acts on $T$. Denote by $\pi$ the Iwasawa projection onto $T$, i.e. $\pi: a n k \mapsto a$. His result is

Theorem $([$ Kos73] $)$. Denote by K.x the orbit of $K$ through $x \in G / K$. Then

$$
\pi(K . x)=\operatorname{conv}(W \cdot x) .
$$

Affine buildings are in many ways similar to symmetric spaces. Often results for symmetric spaces have an analogue in the theory of affine buildings. Some results can be stated simultaniously. Therefore it is a natural question whether a convexity result in the spirit of Kostant's can be found for arbitrary affine buildings $X$. It turns out that an analog statement is true, assuming thickness of $X$. The flat $T$ is replaced by an apartment $A$ (together with a fixed origin 0 ), which is a flat subspace of the affine building $X$, equipped with a natural action of the spherical Weyl group $W$. The role of the Iwasawa projection is played by a retraction of $X$ onto the apartment $A$. The set $K . x$ is, if the automorphism group $\operatorname{Aut}(X)$ is big enough, replaced by the orbit of $x$ under the stabilizer of 0 in $\operatorname{Aut}(X)$.

The main result. We fix an apartment $A$ in $X$. Let $S$ in $A$ be a Weyl chamber, i.e. a translate of the metric closure of a connected component of $A \backslash\left\{H_{i, 0}, i \in I\right\}$ under the affine Weyl group $W^{a}$. The parallel class $\partial S$ of the Weyl chamber $S$ is a chamber in the spherical building $\Delta=\partial X$ at infinity. Let $\mathcal{C}_{f}$ denote the fundamental Weyl chamber. Alcoves in $A$ are translates of a fundamental domain of the action of $W^{a}$. Denote by $\mathbf{c}_{\mathbf{f}}$ a fundamental alcove. 
There are the following two retractions from $X$ onto the fixed apartment $A$ : The canonical retraction $r$ centered at the fundamental alcove $\mathbf{c}_{\mathbf{f}} \in A$ and the retraction $\rho$ centered at $\partial \mathcal{C}_{f}^{\text {op }} \in \partial A$. (See page 4 for definitions.)

Denote by $\bar{\omega}_{i}$ the fundamental weights. For all $i \in I$ and $k \in \mathbb{Z}$ the set $\bar{H}_{i, k}:=\{x \in$ $\left.A \mid\left\langle x^{\vee}, \bar{\omega}_{i}\right\rangle=k\right\}$ is called a dual hyperplane. Usually $W$-convex sets in a building are defined as intersections of finitely many half appartments. Analogously we define a convex set1 to be an intersection of finitely many dual half apartments. The convex hull of a set $Y$, denoted by $\operatorname{conv}(Y)$, is the smallest convex set containing $Y$. Our main result reads as follows

Theorem 3.1. Let $X$ be a thick affine building, $A$ an apartment in $X$. Let $r$ and $\rho$ be defined as above. For all special vertices $x$ in $A$

$$
\rho\left(r^{-1}(W . x)\right)=\operatorname{conv}(W . x) \cap\{\text { vertices } y \in A \text { such that type }(y)=\operatorname{type}(x)\} .
$$

Extending the maps $\rho$ and $r$ to galleries in $X$ starting at 0 , the proof of the theorem can be reduced to the following two facts:

Proposition 3.2. All vertices in $\operatorname{conv}(W . x)$ of the same type as $x$ are endpoints of positively folded galleries of fixed type $t$, where $t$ is the type of a minimal gallery connecting the origin 0 with $x$. All such endpoints are contained in this set.

Proposition 3.3. Every positively folded gallery in the proposition above has a preimage under $\rho$ which is a minimal gallery starting at 0 .

In [GL05] Gaussent and Littelmann study irreducible highest weight representations of semisimple complex or compact Lie groups. They establish a character formula in terms of positively folded galleries. Proposition 3.2 is an easy consequence of their character formula. Note that the first proposition is just a statement about affine Coxeter complexes. One therefore is completely independent of the ambient building. Our main result holds for all thick affine buildings, which need not be associated to an algebraic group, even though the proof relies on deep results about representations of algebraic groups.

An application. Let $X, A$, and $\mathcal{C}_{f}^{\text {op }}$ be as above. Assume $G$ is a group acting on $X$ by automorphisms. Assume further that $G$ has an affine $B N$-pair such that $B$ splits as $B=U T$. Let $K$ be the stabilizer of 0 in $G$. Then $G$ has an Iwasawa decomposition $G=U T K$, where $U$ is the unipotent radical and $T$ is the group of translations in $A$. Therefore we can identify special vertices in $X$ with cosets $u t K$ in $G$, where $u \in U$ and $t \in T$. The origin 0 corresponds to $K$. It is easy to verify that $\rho$ is the map $u t K \mapsto t K$. The set $r^{-1}(W . x)$ is the same as the $K$-orbit of $x$, for all $x \in A$. Note that elements in $A$ are of the form $t K$ for some $t \in T$. The following corollary is just a reformulation of our main result.

Corollary 1.1. Given $x=t K \in X$ the following is true:

$$
\begin{gathered}
\rho(K t K)=\operatorname{conv}(t K) . \\
\text { Or, since } \rho^{-1}\left(t^{\prime} K\right)=U t^{\prime} K, \text { equivalently } \\
\emptyset \neq U t^{\prime} K \cap K t K \Leftrightarrow t^{\prime} K \in \operatorname{conv}(t K) .
\end{gathered}
$$

\footnotetext{
${ }^{1}$ One could also use the somewhat redundant name dual convexity.
} 
The proof of " $\Rightarrow$ " is well known and can be found in BT72]. For partial results on $" \Leftarrow "$ and related questions see for example [GL05, MV00]. Denote by $M$ the matrix describing the Satake isomorphism in terms of natural bases of source and target. Sometimes the implication from the right to the left is stated in terms of positivity of the coefficients of $M$. Rapoport proved such a positivity result in Rap00 which was generalized by Schwer in [Sch06]. Compare also Silberger, Sil75], who proved the equivalence $\emptyset \neq U t^{\prime} K \cap K t K \Leftrightarrow t^{\prime} K \in \operatorname{conv}(t K)$ for algebraic groups defined over $p$-adic fields.

The paper is organized as follows. We set notations and state the needed definition and results in Section 2. Here we also define positively folded galleries, convexity and state the character formula mentioned above. Section 3 is devoted to the proof of our main result. In the last Section the application is shown.

The author would like to thank L. Kramer for suggesting the problem, reading an ealier version of this and for several helpful comments and Christoph Schwer for pointing out the work of Gaussent and Littelmann.

This is part of the author's Ph.d. thesis, written under the supervision of L. Kramer and R. Weiss at the University of Münster, Germany. During the preparation of this paper the author was supported by the Studienstiftung des deutschen Volkes and the $D F G$.

\section{Preliminaries, Definitions And nOtation}

Alcoves and Weyl chambers. For the following definitions see for example Bou02, Bro89. Let $V$ be a vector space over $\mathbb{R}$ and denote by $V^{*}$ its dual. We denote by $\left\langle\alpha, \beta^{\vee}\right\rangle$ the evaluation of $\beta \in V^{*}$ on $\alpha \in V$. Recall the following

Definition 2.1. A subset $R$ of $V$ is a root system in $V$ if the following holds

- $R$ is finite, $0 \notin R$ and $R$ generates $V$

- for all $\alpha \in R$ exists $\alpha^{\vee} \in V^{*}$ such that $\left\langle\alpha, \alpha^{\vee}\right\rangle=2$

- the reflection $r_{\alpha, \alpha} \vee: x \mapsto x-\left\langle\alpha, \alpha^{\vee}\right\rangle \alpha$ leaves $R$ invariant

- for all $\alpha \in R$ the set $\alpha(R)$ is contained in $\mathbb{Z}$.

Note that $R^{\vee}=\left\{\alpha^{\vee}: \alpha \in R\right\}$ is a root system in $V^{*}$. We refer to $R^{\vee}$ as the inverse root system. Is $B$ a basis of $R$ so is $B^{\vee}=\left\{\alpha^{\vee}\right\}_{\alpha \in B}$ a basis of $R^{\vee}$. The elements of the dual basis $\left\{\bar{\omega}_{\alpha}\right\}_{\alpha \in B}$ of $B^{\vee}$ in $V$ are called fundamental weights.

Let $X$ be an (irreducible, simplicial) affine building with apartment system $\mathcal{A}$. Denote by $R$ the associated irreducible root system contained in $V$. Let $B=\left\{\alpha_{i}, i \in I\right\}$ be the root basis of $R$. Further let $W^{a}$ denote the affine and $W$ the spherical Weyl group.

Let $E$ denote the affine space underlying the vector space $V$ on which $R$ is defined. The hyperplane associated to $\alpha_{i} \in B$ is $H_{i, 0}=\left\{x \in E:\left\langle x, \alpha_{i}^{\vee}\right\rangle=0\right\}$. By Weyl chambers we mean translates of the closures of connected components of $E \backslash\left\{H_{i, 0}, i \in I\right\}$ under the affine Weyl group $W^{a}$.

A general hyperplane is an image of some $H_{i, 0}$ under $W^{a}$. For each hyperplane $H$ there exist $\alpha \in R$ and $k \in \mathbb{Z}$ such that $H=H_{\alpha, k}:=\left\{x \in E \mid\left\langle x, \alpha^{\vee}\right\rangle=k\right\}$. We will refer to closures of connected components of $E \backslash\left\{H_{\alpha, k}, \alpha \in R, k \in \mathbb{Z}\right\}$ as alcoves. Denote by 
$\alpha_{0}$ the highest root in $R$. Then $\mathbf{c}_{\mathbf{f}}=\left\{x \in E:\left\langle x, \alpha_{i}^{\vee}\right\rangle \geq 0\right.$ for all $i \in I$, and $\left.\left\langle x, \alpha_{0}^{\vee}\right\rangle\right\}$ is a fundamental alcove. The parallel class $\partial S$ of the Weyl chamber $S$ is an alcove in the spherical building $\Delta=\partial X$ at infinity. A half appartment $H_{\alpha, k}^{ \pm}$is one of the two half spaces $\left\{x \in E:\left\langle x, \alpha^{\vee}\right\rangle \geq k\right\}$ and $\left\{x \in E:\left\langle x, \alpha^{\vee}\right\rangle \leq k\right\}$. Let $S$ be a Weyl chamber. The intersection of all faces of $S$ is the vertex of $S$. The set of Weyl chambers in $E$ sharing the same vertex is in one-to-one correspondence with the chambers of the spherical Coxeter complex associated to $W$.

Denote by $\mathcal{C}_{f}$ the fundamental Weyl chamber. By definition

$$
\begin{aligned}
\mathcal{C}_{f} & =\left\{x \in E \mid\left\langle\alpha_{i}^{\vee}, x\right\rangle \geq 0 \text { for all } i \in I\right\} \\
& =\left\{x \in E \mid x=\sum_{i \in I} k_{i} \bar{\omega}_{i} \text { with } k_{i} \geq 0 \text { for all } i \in I\right\} .
\end{aligned}
$$

Cones and dual cones. The positive cone $\mathcal{C}_{p}$, which is the dual cone of $\mathcal{C}_{f}$, will play an important role in the definition of the convexity we use in the formulation of our main result.

The positive cone $\mathcal{C}_{p}$ can be described as follows:

$$
\begin{aligned}
\mathcal{C}_{p} & =\left\{x \in E \mid\left\langle\bar{\omega}_{i}, x^{\vee}\right\rangle \geq 0 \text { for all } i \in I\right\} \\
& =\left\{x \in E \mid x=\sum_{i \in I} k_{i} \alpha_{i} \text { with } k_{i} \geq 0 \text { for all } i \in I\right\} .
\end{aligned}
$$

Retractions. From now on fix an apartment $A$ in $X$ and an origin 0 in $A$, which is a special vertex. There are two natural retractions from $X$ onto $A$, which can be characterized as follows:

Definition 2.2. Let $c$ be an alcove in $A$. The canonical retraction onto $A$ centered at $c$, denoted by $r_{A, c}: X \rightarrow A$, is characterized by the following two properties:

(1) The alcove $c$ is fixed pointwise by $r_{A, c}$ and each apartment that contains $c$ is mapped isomorphically onto A.

(2) The map $r_{A, c}$ is distance diminishing on $X$.

Definition 2.3. Let $S$ be a Weyl chamber in $A$. The retraction onto $A$ centered at $\partial S$, which will be denoted by $\rho_{A, S}: X \rightarrow A$, is the unique map such that the following conditions are satisfied:

(1) For each apartment $B$ containing a sub-Weyl chamber of $S$ the restriction of $\rho_{A, S}$ to $B$ is an isomorphism onto $A$ fixing $A \cap B$ pointwise.

(2) The map $\rho_{A, S}$ diminishes distances in $X$.

One has the following connection between these two retractions

Theorem ([Bro89], p.171). Let $\rho_{A, S}$ be the retraction onto $A$ centered at $\partial S$ in $A$. Given an alcove $d \in X$ there exists an alcove $c \in A$ such that

$$
\rho_{S, A}(d)=r_{A, c}(d) .
$$

We identify $W$ with $\operatorname{Stab}_{W^{a}}(0)$. The Weyl chamber opposite $\mathcal{C}_{f}$ in $A$ is denoted by $\mathcal{C}_{f}^{\text {op }}$. For simplicity of notation, we write $r$ instead of $r_{A, \mathbf{c}_{\mathbf{f}}}$ and let $\rho$ stand for $\rho_{A, \mathcal{C}_{f}^{\text {op }}}$. 
Choosing apartments $A_{1}, A_{2}$ such that $\partial \mathcal{C}_{f}^{\text {op }} \subset \partial A_{i}$ for $i=1$ and 2 , the corresponding retractions $\rho_{A_{1}, \mathcal{C}_{f}^{\text {op }}}$ and $\rho_{A_{2}, \mathcal{C}_{f}^{\text {op }}}$ are compatible in the sense of the following lemma.

Lemma 2.4. Given apartments $A_{i}, i=1, \ldots, n$, such that $\partial \mathcal{C}_{f}^{\mathrm{op}} \subset \partial A_{i}$ for all $i$. Denote by $\rho_{i}$ the retraction $\rho_{A_{i}, \mathcal{C}_{f}^{\text {op }}}$. Then

$$
\left(\rho_{1} \circ \rho_{2} \circ \ldots \circ \rho_{n}\right)=\rho_{1} .
$$

Proof. For all $i$ the retraction $\rho_{i}$ maps apartments containing representatives of $\partial \mathcal{C}_{f}^{\text {op }}$ isomorphically onto $A$. As a set $X$ equals the union of all such apartments. Every such apartment is isomorphically mapped onto the apartment $A_{1}$ by $\widetilde{\rho}:=\rho_{1} \circ \rho_{2} \circ \ldots \circ \rho_{n}$. A second property of the map $\widetilde{\rho}$ is that it is distance diminishing, since each $\rho_{i}$ is. Therefore $\widetilde{\rho}: X \mapsto A_{1}$ is the unique map defined by these two properties, which is $\rho_{1}$.

For further details see for example [Bro89].

Positively folded galleries. To be able to control how galleries of a given type are stammering, we introduce the slightly more general notion of combinatorial galleries. One can think of a combinatorial gallery as a gallery in the usual sense together with a fixed type.

Definition 2.5. A combinatorial gallery $\gamma$ in $X$ is a sequence of faces and alcoves

$$
\gamma=\left(c_{0}^{\prime} \subset c_{0} \supset c_{1}^{\prime} \subset c_{1} \supset c_{2}^{\prime} \subset \ldots \supset c_{n}^{\prime} \subset c_{n} \supset c_{n+1}^{\prime}\right)
$$

such that

- the source $c_{0}^{\prime}$ and the target (or endpoint) $c_{n+1}^{\prime}$ are vertices in $X$,

- $c_{i}$ is an alcove, for all $i=0 \ldots n$, and

- $c_{i}^{\prime}$ is a codimension one face of $c_{i-1}$ and $c_{i}$ for all $i=1 \ldots n-1$.

The type of a combinatorial gallery $\gamma$ is the list $t=\left(t_{0}, t_{1}, \ldots, t_{n}\right)$ of types $t_{i}$ of the faces $c_{i}^{\prime}$. We say $\gamma$ is minimal if it is the shortest gallery with source $c_{0}^{\prime}$ and target $c_{n+1}^{\prime}$.

We write $\gamma: x \rightsquigarrow y$ if $\gamma$ is a combinatorial gallery with source $x$ and target $y$. If nothing else is specified we mean by gallery a combinatorial gallery in the sense of Definition 2.5.

We fix a type $t$ and denote by $\hat{\mathcal{G}}_{t}$ the set of all minimal galleries of type $t$ in $X$ with source $0 \in A$. We denote by $\mathcal{G}_{t}$ the set of targets $\operatorname{wgt}(\gamma)$ of galleries $\gamma$ in $\hat{\mathcal{G}}_{t}$. This leads to the following

Lemma 2.6. Let $A$ and 0 be as above. Assume that $x$ a special vertex in $A$ and $\gamma: 0 \rightsquigarrow x$ minimal of type $t$. Then

$$
r^{-1}(W . x)=\mathcal{G}_{t} .
$$

Assume $K:=\operatorname{Stab}_{\mathrm{Aut}(\mathrm{X})}(0)$ acts transitive on the set of all apartments containing 0 (e.g. assume $G$ has an affine $B N$-pair), then

$$
r^{-1}(W . x)=K . x .
$$

Proof. The retraction $r$ preserves adjacency relations and distance to 0 in $X$. Part one is an easy consequence of these properties. In part two the required transitivity directly implies that $\mathcal{G}_{t}=K . x$. 
The following definition will help us to describe the set $\rho\left(r^{-1}(W \cdot x)\right)$.

Definition 2.7. Let $H$ be a hyperplane, $d$ an alcove and $S$ a Weyl chamber in the fixed apartment $A$. We say $H$ separates $d$ and $S$ if there exists a representative $S^{\prime}$ of $\partial S$ in A such that $S^{\prime}$ and d are contained in different half spaces determined by $H$.

Definition 2.8. A gallery $\gamma=\left(c_{0}^{\prime} \subset c_{0} \supset c_{1}^{\prime} \subset c_{1} \supset c_{2}^{\prime} \subset \ldots \supset c_{n}^{\prime} \subset c_{n} \supset c_{n+1}^{\prime}\right)$ is positively folded at $i$ if

- $c_{i}=c_{i-1}$ and

- the hyperplane $H:=\operatorname{span}\left(c_{i}^{\prime}\right)$ separates $c_{i}$ and $\mathcal{C}_{f}^{\mathrm{op}}$.

A gallery $\gamma$ is positively folded if it is positively folded at $i$ whenever $c_{i-1}=c_{i}$.

The picture to have in mind is the following: Let $\gamma=\left(x \subset c_{0} \supset c_{1}^{\prime} \subset c_{1} \supset \ldots \subset c_{n} \supset y\right)$ be a positively folded gallery of type $t$. Assume that there exists exactly one folding and let $i_{0}$ denote the associated index. Denote by $\widetilde{\gamma}=\left(x \subset d_{0} \supset d_{1}^{\prime} \subset d_{1} \supset \ldots \subset\right.$ $\left.d_{n} \supset d_{n+1}^{\prime}\right)$ the minimal gallery of the same type $t$ such that $c_{i}=d_{i}$ and $c_{i}^{\prime}=d_{i}^{\prime}$ for all $i=0 \ldots i_{0}-1$. Then $\gamma$ is obtained from $\widetilde{\gamma}$ by successively reflecting "the rest" $\left(d_{i_{0}} \supset d_{i_{0}+1}^{\prime} \subset \ldots \subset d_{n} \supset d_{n+1}^{\prime}\right)$ of $\widetilde{\gamma}$ at the hyperplane $H_{i_{0}}$.

Lemma 2.9. Let $\gamma \in \hat{\mathcal{G}}_{t}$ then $\hat{\rho}(\gamma)$ is a positively folded gallery of the same type as $\gamma$.

Proof. Let $\gamma=\left(x \subset c_{0} \supset c_{1}^{\prime} \subset c_{1} \supset \ldots \subset c_{n} \supset y\right)$ be an image under $\hat{\rho}$ of an element $\sigma \in \hat{\mathcal{G}}_{t}$. Retractions preserve adjacency and hence the type of a gallery. Let $i$ be the smallest index such that $c_{i-1}=c_{i}$ and assume $\gamma$ is not positively folded at $i$. We may assume further that $\sigma$ has already been retracted onto $\gamma$ up to $i-1$, meaning

$$
\sigma=\left(x \subset c_{0} \supset c_{1}^{\prime} \subset c_{1} \supset \ldots \subset c_{i-1} \supset c_{i}^{\prime} \subset d_{i} \supset d_{i+1}^{\prime} \subset \ldots \subset d_{n} \supset y^{\prime}\right),
$$

where $d_{j} \notin A$ for all $j$. There is an apartment $A^{\prime}$ containing $\mathcal{C}_{f}^{\text {op }}, c_{i-1}$ and $d_{i}$. Denote by $H$ the hyperplane in $A^{\prime}$ containing $c_{i}^{\prime}$ and separating $d_{i}$ and $c_{i-1}$. One can find an alcove $b \in \mathcal{C}_{f}^{\text {op }} \in A$ such that $\rho\left(c_{i}\right)=r_{b, A}\left(c_{i}\right)$. The retraction $r_{b, A}$ preserves distance to $b$ and maps $A^{\prime}$ isometrically onto $A$. Hence the wall $H$ separates $c_{i}$ from $r_{b, A}\left(d_{i}\right)$ and also $r_{b, A}\left(d_{i}\right)$ from $\mathcal{C}_{f}^{\mathrm{op}}$. But this is not possible since we assumed that $r_{b, A}\left(d_{i}\right)=c_{i}=c_{i-1}$ and that $c_{i}$ is not separated from $\mathcal{C}_{f}^{\text {op }}$ by $H$.

Dual convexity. Usually a subset $C$ of an apartment $A$ is defined to be $W$-convex if it is the intersection of finitely many half apartments. The $W$ - convex hull of a set $C$ is the intersection of all half apartments containing $C$. The following example illustrates why in our situation this is not the right notion of convexity.

Example 2.10. Let $A$ be a Coxeter Complex of type $\widetilde{A}_{2}$. Let $\alpha_{1}$ and $\alpha_{2}$ be the root basis of the underlying root system $R$. Define $x$ to be the sum $3 \alpha_{1}+3 \alpha_{2}$. Observe that the $W$ convex hull of the Weyl group orbit W.x contains $4 \alpha_{1}+2 \alpha_{2}=: y$. For special vertices $a, b$ denote by $\delta(a, b)$ the length of the shortest gallery $\gamma$ such that a is contained in the first and $b$ contained in the last chamber of $\gamma$. Then $\delta(0, x)=10$ and $\delta(0, y)=11$.

For all elements $z$ in $\rho\left(r^{-1}(W . x)\right)$ the distance $\delta(0, z) \leq 10$, since the two retractions are distance diminishing. Hence $y$ can not be contained in $\rho\left(r^{-1}(W . x)\right)$.

We therefore define a new notion of convexity using hyperplanes dual to the usual ones. 
Definition 2.11. A dual hyperplane is a set $H_{i, k}^{*}=\left\{x \in E \mid\left\langle\bar{\omega}_{\alpha_{i}}, x^{\vee}\right\rangle=k\right\}$, where $k \in \mathbb{Z}$ and $i \in I \cup\{0\}$.

Dual hyperplanes determine dual half apartments. As $H_{i, k}$ is perpendicular to $\alpha$, so is $H_{i, k}^{*}$ perpendicular to $\bar{\omega}_{i}$.

Definition 2.12. A convex set is an intersection of finitely many dual half apartments in $A$, where the empty intersection is defined to be $A$. The convex hull of a set $C$, denoted by $\operatorname{conv}(C)$, is the intersection of all dual half apartments containing $C$.

Let $C$, in the definition above, be an orbit $W \cdot x$ of a vertex $x$ in the fixed apartment $A$. Then one can prove that $\operatorname{conv}(C)$ coincides with the metric convex hull of $W . x$ in $A$.

The following Lemma, which is analogous to [Kos73, Lemma 3.3(2)], plays a crucial role in the proof of our main result. Let $A^{\text {type }}(x)$ denote the set of all vertices in $\operatorname{conv}(W . x)$ having the same type as $x$.

Lemma 2.13. Let $x$ be a special vertex in E. For any special vertex $z$ denote by $z^{+}$ the unique element of $W . z$ that is contained in $\mathcal{C}_{f}$. Then

$$
A^{\text {type }}(x)=\left\{y \in E: x^{+}-y^{+} \in \mathcal{C}_{p} \text { and } \operatorname{type}(x)=\operatorname{type}(y)\right\} .
$$

Proof. W.l.o.g. we may assume that $x \in \mathcal{C}_{f}$. The set $A^{\text {type }}(x)$ is by definition $W$ invariant. It is sufficient to prove that $A^{\text {type }}(x) \cap \mathcal{C}_{f}$ equals the set

$$
\left\{y \in \mathcal{C}_{f}: x-y \in \mathcal{C}_{p} \text { and type }(x)=\operatorname{type}(y)\right\} .
$$

Let $k_{i}=\left\langle\bar{\omega}_{i}, x^{\vee}\right\rangle$, then $x=\sum k_{i} \bar{\omega}_{i}$. Denote by $t$ the type of $x$. By the definition of dual hyperplanes

$$
A^{\text {type }}(x) \cap \mathcal{C}_{f}=\mathcal{C}_{f} \cap\left(\mathcal{C}_{p}^{\mathrm{op}}+x\right)=\left(\bigcap_{i=1}^{n} H_{i, 0}^{+}\right) \cap\left(\bigcap_{i=1}^{n}\left(H_{i, k_{i}}^{*}\right)^{-}\right) .
$$

Let $y$ be an element of $A^{\text {type }}(x) \cap \mathcal{C}_{f}$. From (11) we deduce $\left\langle\bar{\omega}_{i}, y^{\vee}\right\rangle \leq\left\langle\bar{\omega}_{i}, x^{\vee}\right\rangle=k_{i}$ for all $i \in I$. Consequently

$$
\left\langle\bar{\omega}_{i},(x-y)^{\vee}\right\rangle=\left\langle\bar{\omega}_{i}, x \vee\right\rangle-\left\langle\bar{\omega}_{i}, y^{\vee}\right\rangle \geq 0 \Longleftrightarrow x-y \in \mathcal{C}_{p} .
$$

Conversely assume that $y \in \mathcal{C}_{f}$ and $x-y \in \mathcal{C}_{p}$. Again one has

$$
0 \leq\left\langle\bar{\omega}_{i},(x-y)^{\vee}\right\rangle=\left\langle\bar{\omega}_{i}, x^{\vee}\right\rangle-\left\langle\bar{\omega}_{i}, y^{\vee}\right\rangle
$$

and hence $\left\langle\bar{\omega}_{i}, x^{\vee}\right\rangle \geq\left\langle\bar{\omega}_{i}, y^{\vee}\right\rangle$. Therefore $y \in A^{\text {type }}(x)$.

A character formula. We use this section to fix notation and state the character formula we will use in the proof of our main result. For further references on representation theory see for example [Bou05] or [Hum72]. For references on the construction of $\mathcal{I}^{a}$ (defined below) see for example [BT72].

Let $G$ be a connected semisimple complex algebraic group. Let $T$ be a maximal torus in $G$. Denote by $\mathfrak{X}$ the character group of $T$ and the subset of dominant characters in $\mathfrak{X}$ by $\mathfrak{X}_{+}$. Apartments in the affine building $\mathcal{I}^{a}$ associated to $G(\mathbb{C} \llbracket t \rrbracket)$ can be identified with translates $g A$ of $A:=\mathfrak{X} \otimes_{\mathbb{Z}} \mathbb{R}$. Dominant weights $\lambda \in \mathfrak{X}_{+}$correspond to special vertices in $\mathcal{C}_{f}$. Denote by $V_{\lambda}$ the irreducible complex representation of highest weight $\lambda$ for $G$. Let $\chi_{\lambda}$ be the character of $V_{\lambda}$. 
In this setting Gaussent and Littelmann proved the following character formula. Note that our root system $R$ actually is the cooroot system for the one considered in [GL05]. Accordingly we switch cocharacters to characters, etc.

Theorem 2.14. GL05] Fix $\lambda \in \mathfrak{X}_{+}$. Then

$$
\sum_{\gamma \in L S(t)} k^{\mathrm{wgt}(\gamma)}=\chi_{y}=\sum_{\nu \in X} p_{\nu} k^{\nu}
$$

where $p_{\nu}$ is the dimensions of the $v$-weightspace of $V_{\lambda}$ and $\operatorname{wgt}(\gamma)$ denotes the endpoint of $\gamma$.

The set $L S(t)$, the so called $L S$-galleries, is a certain subset of the set of positively folded galleries in the fixed apartment $A$ that start at 0 and are of fixed type $t$. The term $k^{w g t(\gamma)}$ in the formula is a group like element of the group algebra associated to the targets $\operatorname{wgt}(\gamma)$ of $L S$-galleries. For all $\nu \in \mathcal{C}_{f}$ the coefficients $p_{\lambda, \nu}$ are strictly positive if and only if $\nu \leq \lambda$.

Remark 2.15. GL05 use a slightly more general notion of (combinatorial) galleries as given in Definition 2.5. Nevertheless their result is true in our setting as well, which follows by results in Sch06].

\section{The MAIN RESUlT}

This section is devoted to the proof of our main result:

Theorem 3.1. Let $X$ be a thick affine building with fixed apartment $A$. Let $x$ be a special vertex in $A$. Then

$$
\begin{aligned}
\rho\left(r^{-1}(W . x)\right) & =\operatorname{conv}(W . x) \cap\{\text { vertices } y \in A \text { of type } \operatorname{type}(x)\} \\
& =A^{\text {type }}(x) .
\end{aligned}
$$

Extending the retractions $\rho$ and $r$ to minimal galleries in $X$ starting at 0 , the proof of Theorem 3.1 can be reduced to the following two facts:

Proposition 3.2. Let $X, A$ and $x$ be as in 3.1. Denote by $x^{+}$the unique element of $W . x$ contained in $\mathcal{C}_{f}$. Let $\gamma: \rightsquigarrow x^{+}$be a minimal gallery and denote by $t$ its type. All vertices in $A^{\text {type }}(x)=\{y \in \operatorname{conv}(W . x)$ : such that type $(x)=$ type $(y)\}$ are targets of positively folded galleries of type $t$. Targets of such galleries are contained in $A^{\text {type }}(x)$.

Proposition 3.3. Every positively folded gallery in Proposition 3.2 has a pre-image under $\rho$ which is a minimal gallery starting at 0 .

With these propositions by hand, the proof of the main result reads as follows:

Proof of [3.1. By Lemma 2.6, the set $\left.r^{-1}(W . x)\right)=\mathcal{G}_{t}$. Hence $\left.\rho\left(r^{-1}(W . x)\right)\right)=\rho\left(\mathcal{G}_{t}\right)$. Denote by $\hat{r}$ and $\hat{\rho}$ the extensions of $r$ and $\rho$ to minimal galleries starting at 0 . Lemma 2.9 implies that $\hat{\rho}\left(\hat{\mathcal{G}}_{t}\right)$ is a set of positively folded galleries in $A$, thus $\rho\left(\mathcal{G}_{t}\right)$ is the set of targets of the galleries in $\hat{\rho}\left(\hat{\mathcal{G}}_{t}\right)$. By Propositions 3.2 and 3.3 the assertion holds.

It remains to prove Propositions 3.2 and 3.3 . 
Proof of 3.2. Denote by $\Gamma_{t}^{+}$be the set of all positively folded galleries of type $t$ in $A$ starting in 0 . This set contains $L S(t)$, the so called $L S$-galleries (see GL05] for definition). Let $G$ be a connected semisimple complex algebraic group $G$, denote by $\mathfrak{X}$ the character group of a chosen maximal torus $T$. Let $G$ be chosen such that $A$ can be identified with $\mathfrak{X} \otimes_{\mathbb{Z}} \mathbb{R}$. By Theorem 2.14 we have the following formula for $\chi_{x^{+}}$, the (irreducible) highest weight representation $V_{x^{+}}$with highest weight $x^{+}$:

$$
\sum_{\sigma \in L S(t)} k^{\mathrm{wgt}(\sigma)}=\chi_{x^{+}}=\sum_{\nu \in X} p_{\nu} k^{\nu}
$$

where $\operatorname{wgt}(\sigma)$ is the target of $\sigma$. The coefficients $p_{\nu}$ in the right part are the dimension of the $\nu$-weight space of $V_{x^{+}}$, which are positive if and only if $\nu \leq x^{+}$. Simply comparing coefficients of $k^{\nu}$ on the left and right hand side of the equation there has to exist an $L S$-gallery with target $\nu$ for all $\nu \leq x^{+}$. By Lemma 2.13 the theorem follows.

Proof of 3.3. Let $\gamma=\left(0 \subset c_{0} \supset d_{1} \subset c_{1} \supset d_{2} \subset \ldots \supset d_{n} \subset c_{n} \supset y\right)$ be a positively folded gallery of type $t$ which is contained in $A$. We will inductively construct a preimage of $\gamma$ under $\rho$ which is minimal in $X$. Denote by $J \subset\{1,2, \ldots, n\}$ the set of indices $\left\{i_{1}, \ldots, i_{k}\right\}$ at which $\gamma$ is folded. Hence for each $i \in J$ we have that $c_{i_{j}}=c_{i_{j}-1}$. Assume $i_{1}<i_{2}<\ldots<i_{k}$. Let $H_{i_{1}}$ be the hyperplane in $A$ separating $c_{i_{1}}$ from $\mathcal{C}_{f}^{\text {op }}$. We write $H_{i_{1}}^{-}$(respectively $H_{i_{1}}^{+}$) for the half space of $A$ determined by $H_{i_{1}}$ containing a subsector of $\mathcal{C}_{f}^{\text {op }}$ (respectively a subsector of $\mathcal{C}_{f}$ ). Since $X$ is a thick building there exists an apartment $A_{1}$ such that $A_{1} \cap A=H_{i_{1}}^{-}$.

Define $A^{\prime}:=H_{i_{1}}^{+} \cup\left(A_{1} \backslash A\right)$. By construction $\gamma$ is contained in $A^{\prime}$. Let $r_{i_{1}}$ be the reflection associated to $H_{i_{1}}$ in $A^{\prime}$ and define $\gamma^{1}$ as follows:

$$
\gamma^{1}=\left(0 \subset c_{0} \supset d_{1} \subset \ldots \supset d_{i_{1}} \subset c_{i_{1}}^{1} \supset d_{i_{1}+1}^{1} \subset \ldots \supset d_{n}^{1} \subset c_{n}^{1} \supset y^{1}\right)
$$

where $c_{j}^{1}=r_{i_{1}}\left(c_{j}\right)$ and $d_{j}^{1}=r_{i_{1}}\left(d_{j}\right)$ for all $j \geq i_{1}$. Note that $d_{i_{1}}=d_{i_{1}}^{1}$ since $d_{i_{1}}$ is contained in $H_{i_{1}}$. Then $\gamma^{1}$ is a gallery of the same type as $\gamma$ which is not folded at $i_{1}$. Furthermore $\gamma^{1}$ is minimal up to the next folding index, i.e. the shortened gallery $\left(0 \subset c_{0} \supset d_{1} \subset \ldots \supset d_{i_{1}} \subset c_{i_{1}}^{1} \supset d_{i_{1}+1}^{1} \subset \ldots \supset d_{i_{2}-1}^{1} \subset c_{i_{2}-1}^{1}\right)$ is minimal. This step reduced the number of folding indices by one. The construction also directly implies that $\rho\left(\gamma^{1}\right)=\gamma$.

We shorten the gallery $\gamma$ to

$$
\gamma_{1}=\left(x^{1} \subset c_{i_{1}}^{1} \supset d_{i_{1}+1}^{1} \subset \ldots \supset d_{n}^{1} \subset c_{n}^{1} \supset y^{1}\right)
$$

where $x^{1}$ is a vertex of $c_{i_{1}}$ that is chosen such that $\left(x^{1} \subset c_{i_{1}}^{1} \supset d_{i_{1}+1}^{1} \subset \ldots \supset d_{i_{2}}^{1} \subset c_{i_{2}-1}^{1}\right)$ is a minimal gallery from $x^{1}$ to $c_{i_{2}-1}^{1}$. By definition $\gamma^{1}$ is positively folded of suitably shortened type and contained in $A_{1}$. As in the first step we can construct a gallery

$$
\widetilde{\gamma}^{2}=\left(x_{1} \subset c_{i_{1}}^{1} \supset d_{i_{1}+1}^{1} \ldots \supset d_{i_{2}}^{1} \subset c_{i_{2}}^{2} \supset d_{i_{2}+1}^{2} \subset \ldots \supset d_{n}^{2} \subset c_{n}^{2} \supset y^{2}\right)
$$

having the analogous properties. Denote by $\rho_{1}$ the retraction $\rho_{A_{1}, \mathcal{C}_{f}^{\text {op }}}$ onto $A_{1}$. The construction above implies that $\rho_{1}\left(\widetilde{\gamma}^{2}\right)=\gamma^{1}$.

Iterating the same procedure $k$ times, we get a gallery

$$
\gamma^{k}=\left(0 \subset c_{0} \supset d_{1} \ldots d_{i_{1}} \subset c_{i_{1}}^{1} \supset d_{i_{1}+1}^{1} \ldots \ldots d_{i_{k}}^{k-1} \subset c_{i_{k}}^{k} \supset d_{i_{k}+1}^{k} \ldots d_{n}^{k} \subset c_{n}^{k} \supset y^{k}\right)
$$

and a sequence of apartments $A_{1}, \ldots A_{k}$ such that $c_{*}^{j}, d_{*}^{j} \subset A_{j}$ for all $j=1, \ldots, k$. By construction $\gamma^{k}: 0 \rightsquigarrow y^{k}$ is minimal in $X$ and of the same type as $\gamma$. We refer 
to the retraction $\rho_{A_{j}, \mathcal{C}_{f}^{\text {op }}}$ onto the apartment $A_{j}$ as $\rho_{j}$. Lemma 2.4 then implies, that $\rho\left(\gamma^{k}\right)=\left(\rho_{k} \circ \rho_{k-1} \circ \ldots \circ \rho_{1}\right)(\gamma)$. Hence $\gamma^{k}$ is the desired preimage of $\gamma$.

\section{An APPLiCAtion}

Let $A, X, 0$ be as above, and let $G$ be a group acting on $X$ by automorphisms. Assume that $G$ has an affine $B N$-pair. The group $B$ stabilizes $\mathcal{C}_{f}^{\text {op }}$, where the unipotent radical $U$ is transitive on the set of all apartments containing subsectors of $\mathcal{C}_{f}^{\text {op }}$ and $T$ is the group of $G$ of translations in $A$. Assume further that $B$ splits as $B=U T$. Let $K$ be the stabilizer of 0 in $G$. Then $G$ has an Iwasawa decomposition

$$
G=B K=U T K \text {. }
$$

Special vertices in $X$ are in one-to-one correspondence with cosets of $K$ in $G$ : Let $x$ be a special vertex. There exists an element $u \in U$ such that $\left(u^{-1}\right) \cdot x \in A$. Let $t \in T$ be the translation mapping 0 to $\left(u^{-1}\right)$.x. Identify the origin 0 with $K$, then $x$ corresponds to the coset $u t K$. Let $u$ and $t$ be chosen as above, then:

$$
\begin{aligned}
\text { \{special vertices in } X\} & \stackrel{1: 1}{\longleftrightarrow}\{\text { cosets of } K \text { in } G\} \\
0 & \longmapsto K \\
X \ni x & \longmapsto u t K \text { with } u, t \text { chosen as above. }
\end{aligned}
$$

Note that elements in $A$ correspond to cosets of the form $t K$ with $t \in T$. One can prove that the retraction $\rho$ is the map $u t K \mapsto t K$. For all $t K \in A$ the set $r^{-1}(W . t K)$ is the same as the $K$-orbit of $t K$, i.e. $r^{-1}(W . t K)=K t K$. The following is just a direct reformulation of Theorem 3.1 .

Corollary 4.1. For all $t K \in A$ we have

$$
\begin{gathered}
\rho(K t K)=\operatorname{conv}(t K) \\
\text { or, since } \rho^{-1}\left(t^{\prime} K\right)=U t^{\prime} K \text {, equivalently } \\
\emptyset \neq U t^{\prime} K \cap K t K \Leftrightarrow t^{\prime} K \in \operatorname{conv}(t K) .
\end{gathered}
$$

The proof of " $\Rightarrow "$ in the second statement is well known and can be found in [BT72]. As already mentioned in the introduction partial results on " $\Leftarrow$ " and related questions can, for example, be found in [GL05, MV00, Rap00, Sch06] or [Sil75].

\section{REFERENCES}

[Bou02] N. Bourbaki. Lie groups and Lie algebras. Chapters 4-6. Elements of Mathematics (Berlin). Springer Verlag, Berlin, 2002. Translated from the 1968 French original by Andrew Pressley.

[Bou05] N. Bourbaki. Lie groups and Lie algebras. Chapters 7-9. Elements of Mathematics (Berlin). Springer-Verlag, Berlin, 2005. Translated from the 1975 and 1982 French originals by Andrew Pressley.

[Bro89] K. S. Brown. Buildings. Springer-Verlag, New York, 1989.

[BT72] F. Bruhat und J. Tits. Groupes réductifs sur un corps local. Inst. Hautes Études Sci. Publ. Math., (41):5-251, 1972.

[GL05] S. Gaussent und P. Littelmann. LS galleries, the path model and MV cycles. Duke Math. J., $127(1): 35-88,2005$.

[Hum72] J. E. Humphreys. Introduction to Lie algebras and representation theory. Springer-Verlag, New York, 1972. Graduate Texts in Mathematics, Vol. 9. 
[Kos73] B. Kostant. On convexity, the Weyl group and the Iwasawa decomposition. Ann. Sci. École Norm. Sup. (4), 6:413-455 (1974), 1973.

[MV00] I. Mirković und K. Vilonen. Perverse sheaves on affine Grassmannians and Langlands duality. Math. Res. Lett., 7(1):13-24, 2000.

[Rap00] M. Rapoport. A positivity property of the Satake isomorphism. Manuscripta Math., 101(2):153-166, 2000.

[Sch23] I. Schur. Sitzungsber. Berl. Math. Ges., 22:9-20, 1923.

[Sch06] C. Schwer. Galleries, Hall-Littlewood polynomials, and structure constants of the spherical Hecke algebra. Int. Math. Res. Not., Seiten Art. ID 75395, 31, 2006.

[Sil75] A. J. Silberger. Convexity for a simply connected p-adic group. Bull. Amer. Math. Soc., 81(5):910-912, 1975.

Petra Hitzelberger, Mathematisches Institut der Westfälischen Wilhelms-Universität Münster, Einsteinstrasse 62, 48149 Münster hitzelberger@uni-muenster.de 